\title{
Correction to: Referendums: increasingly unpopular among the 'winners' of modernization? Comparing public support for the use of referendums in Switzerland, the Netherlands, the UK, and Hungary
}

\section{Sebastien Rojon ${ }^{1}$ - Arieke J. Rijken ${ }^{2}$}

Published online: 3 August 2021

(c) Springer Nature Limited 2021

\section{Correction to: Comparative European Politics (2021) 19:49-76 https://doi.org/10.1057/s41295-020-00222-5}

After publication, it was noticed that the funding note has been missed in the original publication of the article. The funding note was added: This research was made possible by two projects financed by the European Research Council (ERC) under the European Union's Horizon 2020 research and innovation programme: POLPART (Grant Agreement No 339829) and POLITICIZE (Grant Agreement No 772695).

Publisher's Note Springer Nature remains neutral with regard to jurisdictional claims in published maps and institutional affiliations.

The original article can be found online at https://doi.org/10.1057/s41295-020-00222-5.

Sebastien Rojon

sebastien.rojon@ulb.ac.be

Arieke J. Rijken

Rijken@nidi.nl

1 Le Centre d'Étude de la Vie Politique, Université Libre de Bruxelles, Campus Solbosch CP124, 44 Avenue Jeanne, 1050 Brussels, Belgium

2 Netherlands Interdisciplinary Demographic Institute, P.O. Box 11650, 2502 AR The Hague, The Netherlands 